\title{
Strongly interacting $p$-wave Fermi gas in two dimensions: Universal relations and breathing mode
}

\author{
Yi-Cai Zhang and Shizhong Zhang \\ Department of Physics and Center of Theoretical and Computational Physics, The University of Hong Kong, Hong Kong, China
}

(Received 29 November 2016; published 6 February 2017)

\begin{abstract}
The contact is an important concept that characterizes the universal properties of a strongly interacting quantum gas. It appears in both thermodynamic (energy, pressure, etc.) and dynamic quantities (radio-frequency and Bragg spectroscopies, etc.) of the system. Very recently, the concept of contact was extended to higher partial waves; in particular, the $p$-wave contacts have been experimentally probed in recent experiments. So far, discussions on $p$-wave contacts have been limited to three dimensions. In this paper, we generalize the $p$-wave contacts to two dimensions and derive a series of universal relations, including the adiabatic relations, high-momentum distribution, virial theorem, and pressure relation. At the high-temperature and low-density limit, we calculate the $p$-wave contacts explicitly using virial expansion. A formula which directly connects the shift of the breathing-mode frequency and the $p$-wave contacts is given in a harmonically trapped system. Finally, we also derive the relationships between interaction parameters in three- and two-dimensional Fermi gases and discuss possible experimental realization of a two-dimensional Fermi gas with $p$-wave interactions.
\end{abstract}

DOI: 10.1103/PhysRevA.95.023603

\section{INTRODUCTION}

In cold atomic gas, the resonance $p$-wave interaction was realized experimentally quite some time ago in both ${ }^{40} \mathrm{~K}$ and ${ }^{6} \mathrm{Li} \mathrm{[1-10].} \mathrm{It} \mathrm{was} \mathrm{observed} \mathrm{that} \mathrm{the} \mathrm{system} \mathrm{in} \mathrm{general}$ suffers significant loss close to resonance, preventing the systematic study of the many-body system in equilibrium and, in particular, the achievement of the superfluid regime. Recently, radio-frequency spectroscopic measurement of ${ }^{40} \mathrm{~K}$ showed that the normal state of a $p$-wave Fermi gas close to resonance can achieve a quasiequilibrium state with equilibration between scattering fermions and the shallow $p$-wave dimers within the $p$-wave centrifugal barrier, thus establishing a strongly interacting $p$-wave system [11]. The extracted free-energy reduction close to resonance is of the order of the Fermi energy. Furthermore, it was shown that just as in the $s$-wave case [12-15], the $p$-wave Fermi gas also has universal relations, except that now both the $p$-wave scattering volume $v$ and the effective range $R$ are relevant. Apart from this, there now appear several molecular states due to the different orientations of the angular momentum quantization of the molecule. As a result, the contact parameters have to be generalized, which leads to a set of universal relations that has been discussed in detail in recent works [16-22]. Explicit calculation of the $p$-wave contact within the Noziéres and Schmitt-Rink formula has been carried out and has generally good agreement with the experimental findings [23].

So far, $p$-wave contacts have been defined and discussed mostly in the three-dimensional case. In two dimensions, the derivation of these universal relations proceeds essentially in the same manner as in three dimensions, except that while in three dimensions, one is dealing with a power-law divergence; in two dimensions, one has to deal with the logarithmic divergence. This requires a slight generalization, especially in dealing with the $p$-wave effective range. Another interesting aspect of two-dimensional systems is the apparent scale invariance in the $s$-wave $\delta$-function interaction [24], which would predict a breathing-mode frequency exactly at twice the trap frequency. Here we investigate a similar problem in two-dimensional (2D) $p$-wave resonance, derive the equation of motion for the breathing mode, and show that the $p$-wave contact is also implicated in the equation of motion. In particular, we show that at resonance, the contact parameter related to the effective range breaks the scale invariance and determines the breathing-mode frequency shift.

In this paper, we extend the concept of $p$-wave contacts to two dimensions. In Sec. II, we review some basic facts about low-energy scattering, in particular, the scattering amplitude for $p$-wave interaction and its associated weakly bound state. In Sec. III, we define the $p$-wave contacts in two dimensions and derive the adiabatic theorem. Universal relations for the tail of momentum distribution, the virial theorem, and the pressure relation are given in Sec. IV. In Sec. V, we give explicit calculation of the two contacts in two special cases: a two-body bound state and high temperature. We apply the theory to the trapped case in Sec. VI and derive the frequency shift of the breathing mode in terms of the $p$-wave contact. A general expression for the frequency shift is obtained, and its explicit evaluation is given at high temperature. We give a summary in Sec. VII. Two appendixes discuss the detailed derivations of the frequency shift of the breathing mode and virial theorem in a trap and the relation between effective $p$-wave scattering parameters in two dimensions and those of three dimensions.

\section{TWO-BODY SCATTERING AND BOUND STATES}

For spinless Fermi gas, the $s$-wave interaction is totally suppressed due to the Pauli principle. So at low energy, it is the $p$-wave scattering channel that dominates. For a short-range potential, as is usually the case in cold-atom experiments, the effective range expansion for a $p$-wave in two dimensions is [25-28]

$$
k^{2}\left[\cot \delta_{1}-\frac{2}{\pi} \ln (\rho k)\right]=-\frac{1}{a}+\frac{1}{2} r_{1} k^{2}+O\left(k^{4}\right),
$$

where $\delta_{1}$ is the $p$-wave phase shift and $r_{1}$ is a dimensionless parameter; $a$ and $\rho$ are the scattering area and effective range, 
respectively. The above equation can be rewritten as

$$
k^{2} \cot \delta_{1}=-\frac{1}{a}+\frac{2 k^{2}}{\pi} \ln (R k)+O\left(k^{4}\right) .
$$

Hereafter, we will refer to $R \equiv \rho \exp \left(\pi r_{1} / 4\right)$ as the $p$-wave effective range.

The Schrödinger equation for the relative motion of the two scattering fermions in two dimensions is given by (setting $\hbar=1$ and mass $M=1$ )

$$
\left[\frac{1}{r} \frac{\partial}{\partial r}\left(r \frac{\partial}{\partial r}\right)+\frac{1}{r^{2}} \frac{\partial^{2}}{\partial \theta^{2}}+k^{2}-U(r)\right] \psi(r, \theta)=0 .
$$

The radial and angular parts of the wave function $\psi(r, \theta)$ can be separated for a central potential $U(r), \psi(r, \theta) \equiv R_{k}(r) T(\theta)$, where $T(\theta)$ satisfies the following equation:

$$
\frac{d^{2} T}{d \theta^{2}}+m^{2} T=0
$$

and its solution is given by

$$
T(\theta)=\frac{1}{\sqrt{2 \pi}} e^{ \pm i m \theta}
$$

where $m \in Z$ is the quantum number of the angular part and $m= \pm 1$ for $p$-wave scattering. The radial part of the wave function satisfies

$$
\frac{1}{r} \frac{d}{d r}\left(r \frac{d R(r)}{d r}\right)+\left[k^{2}-U(r)-\frac{m^{2}}{r^{2}}\right] R_{k}(r)=0 .
$$

Let the range of the potential $U(r)$ be $r_{0}$. Then for $r>r_{0}$, the radial wave function $R_{k}(r)$ can be written as a linear combination of two linearly independent Bessel functions,

$$
R_{k}(r)=\frac{\pi k}{2}\left[\cot \delta_{m}(k) J_{m}(k r)-N_{m}(k r)\right] .
$$

Here $J_{m}$ and $N_{m}$ are the Bessel functions of the first and second kinds. When $r \gg 1 / k$, using the asymptotic expressions for Bessel functions, the wave function $R_{k}(r)$ becomes

$$
R_{k}(r) \propto \frac{1}{2} \sqrt{\frac{2}{\pi k r}}\left[S e^{i\left(k r-\frac{m \pi}{2}-\frac{\pi}{4}\right)}+e^{-i\left(k r-\frac{m \pi}{2}-\frac{\pi}{4}\right)}\right],
$$

where we have defined the $S$ matrix in terms of phase shift $\delta_{m}$,

$$
S \equiv \frac{1+i \tan \delta_{m}}{1-i \tan \delta_{m}}=e^{2 i \delta_{m}} .
$$

The scattering wave function can be written as

$$
\psi=e^{i k x}+\frac{f}{\sqrt{r}} e^{i k r}=e^{i k r \cos \theta}+\frac{f}{\sqrt{r}} e^{i k r},
$$

where $f \equiv \sum \hat{f}_{m} e^{i m \theta} / \sqrt{2 \pi}$ is the scattering amplitude in two dimensions. Taking the $m$ th angular component of the scattering wave function,

$$
\psi_{m}=i^{m} J_{m}(k r) e^{i m \theta}+\frac{\hat{f}_{m}}{\sqrt{r}} e^{i m \theta} e^{i k r},
$$

and comparing with $R_{k}(r)$ when $k r \gg 1$, we get the scattering amplitude of the $m$ th wave as

$$
\hat{f}_{m}=e^{i \pi / 4} \sqrt{\frac{4}{k}} \frac{1}{\cot \delta_{m}(k)-i} .
$$

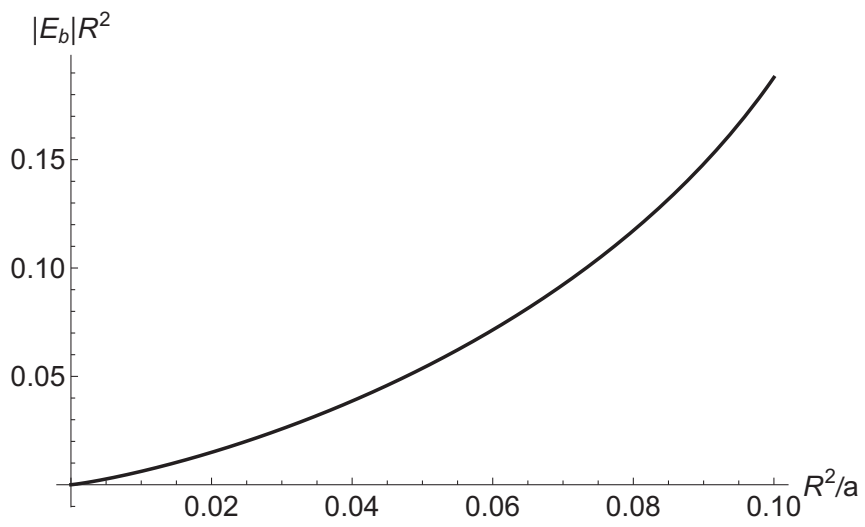

FIG. 1. The variations of binding energy of bound state $\left|E_{b}\right|$ with $1 / a\left(\right.$ fixed $R$ ). Near the resonance $1 / a \rightarrow 0_{+}$, the bound-state energy approaches zero.

For $p$-wave scattering with $m= \pm 1$ in two dimensions, using the effective range expansion equation (2), we obtain

$$
\hat{f}_{ \pm 1}=e^{i \pi / 4} \sqrt{\frac{4}{k}} \frac{k^{2}}{-\frac{1}{a}+\frac{2 k^{2}}{\pi} \ln (R k)-i k^{2}} .
$$

The total scattering cross section (taking into account the degeneracy of $m= \pm 1$ )

$$
\sigma=\int_{0}^{2 \pi}\left[\left|\hat{f}_{-1}\right|^{2}+\left|\hat{f}_{1}\right|^{2}\right] d \theta=\frac{16 \pi \sin ^{2}\left(\delta_{1}\right)}{k} .
$$

As we shall show later, the effective range $R$ is always positive, and as a result, it is possible that a shallow two-body bound state emerges when $a>0$. The binding energy of the shallow two-body bound state must be much smaller than $1 / r_{0}^{2}$ in order to be consistent with effective range expansion. Letting $k=i \kappa$, one finds that the imaginary part in the denominator of Eq. (12) vanishes identically, and the real part is zero when $(a>0)$

$$
x^{2} \ln (x)=-\frac{\pi}{2} \frac{R^{2}}{a},
$$

where $x=R \kappa$ and the bound-state energy is given by $E_{b}=$ $-\kappa^{2}$. The above equation can be solved using Lambert's $W$ function, which is $x=\exp \left[\frac{1}{2} W_{-1}\left(-\pi R^{2} / a\right)\right]$. In the limit when $1 / a \rightarrow 0_{+}$, one finds that $E_{b} \simeq \pi /\left[a \ln \left(\pi R^{2} / a\right)\right]$ and $E_{b}$ tends to $0_{-}$. The other solution of Eq. (14) is of the order of or larger than $1 / r_{0}^{2}$ and has to be discarded. The energy of the shallow bound state is shown in Fig. 1.

At low energy when $k \ll 1 / r_{0}$, the radial wave function $R_{k}$ can be expanded in terms of powers of $k^{2}$, e.g., $R_{k}(r)=$ $u_{0}(r)+k^{2} u_{1}(r)+O\left(k^{4}\right)$, and each satisfies

$$
\begin{gathered}
{\left[-\frac{1}{r} \frac{d}{d r}\left(r \frac{d}{d r}\right)+U(r)+\frac{1}{r^{2}}\right] u_{0}(r)=0,} \\
{\left[-\frac{1}{r} \frac{d}{d r}\left(r \frac{d}{d r}\right)+U(r)+\frac{1}{r^{2}}\right] u_{1}(r)=k^{2} u_{0}(r) .}
\end{gathered}
$$

For $r \gg r_{0}$, the radial wave function $R_{k}(r)$ is given in Eq. (6). Using effective range expansion for the scattering phase shift in Eq. (2) and considering the regime where $r_{0} \ll r \ll 1 / k$, 
the radial wave function, and hence $u_{0,1}(r)$, can be written as

$$
\begin{gathered}
u_{0}(r)=-\frac{1}{r}+\frac{\pi r}{4 a}, \\
u_{1}(r)=\frac{r}{2} \ln \frac{r}{\tilde{R}}-\frac{\pi r^{3}}{32 a},
\end{gathered}
$$

where $\tilde{R}=2 \exp \left(1 / 2-\gamma_{E}\right) R$ and $\gamma_{E} \simeq 0.577$ is the EulerMascheroni constant. At the same time, the wave function near the origin should be regular, which satisfies $R_{k}(r=0)=0$ and $\left.\left(r \partial R_{k} / \partial r\right)\right|_{r=0}=0$ for a not very singular interatomic potential.

Bethe's integral formula for a three-dimensional $s$-wave effective range [29] has been generalized to arbitrary partial waves [30] and arbitrary dimensions [26]. For our twodimensional $p$-wave case, it takes following form:

$$
\int_{0}^{r_{c}} r u_{0}^{2}(r) d r=\ln \frac{e^{\gamma_{E}} r_{c} / 2}{R}-\frac{\pi r_{c}^{2}}{4 a}+\frac{\pi^{2} r_{c}^{4}}{64 a^{2}},
$$

where $r_{c} \gg r_{0}$ is a cutoff length scale. Note that the normalization of the wave function is slightly different (up to a factor $\sqrt{\pi / 2 r}$ ) from that in Ref. [26]. Because the left-hand side of Eq. (19) is positive definite, the effective range is bounded by $0<R<e^{\gamma_{E}} r_{0} / 2 \simeq 0.89 r_{0}$ close to resonance $(a \rightarrow \infty)$. Note that in the following, we will always refer to "resonance" as $1 / a=0$, just as in the three-dimensional case where the $s$-wave scattering length $1 / a_{s} \rightarrow 0$. Bethe's formula for effective range [Eq. (19)] will play an important role in the derivation of the breathing-mode frequency shift in Sec. VI.

Before we end this section, we would like to derive two relations that relate the change of scattering parameters to the variation of interatomic potential $U(r) \rightarrow U(r)+\delta U(r)$. As in the case of three-dimensional $p$-wave scattering [17], two important relations related to the scattering parameters ( $a$ and $R$ ) can be obtained:

$$
\begin{gathered}
\delta(1 / a)=-\frac{2}{\pi} \int_{0}^{\infty} r \delta(U) u_{0}^{2}(r) d r \\
\delta(\ln R)=2 \int_{0}^{\infty} r \delta(U) u_{0}(r) u_{1}(r) d r .
\end{gathered}
$$

\section{DEFINING THE $p$-WAVE CONTACTS}

To define the $p$-wave contacts in two dimensions, we follow the route of Ref. [17]. Let us consider the two-body density matrix

$$
\begin{aligned}
\rho_{2}\left(\vec{r}_{1}, \vec{r}_{2} ; \vec{r}_{2}^{\prime}, \vec{r}_{1}^{\prime}\right) & \equiv\left\langle\psi^{\dagger}\left(\vec{r}_{1}\right) \psi^{\dagger}\left(\vec{r}_{2}\right) \psi\left(\vec{r}_{2}^{\prime}\right) \psi\left(\vec{r}_{1}^{\prime}\right)\right\rangle \\
& =\rho_{2}^{*}\left(\vec{r}_{1}^{\prime}, \vec{r}_{2}^{\prime} ; \vec{r}_{2}, \vec{r}_{1}\right),
\end{aligned}
$$

written in terms of second-quantized field operators $\psi . \rho_{2}$ is a Hermitian matrix, so it can be diagonalized,

$$
\rho_{2}\left(\vec{r}_{1}, \vec{r}_{2} ; \vec{r}_{2}^{\prime}, \vec{r}_{1}^{\prime}\right)=\sum_{i} n_{i} \phi_{i}^{*}\left(\vec{r}_{1}, \vec{r}_{2}\right) \phi_{i}\left(\vec{r}_{1}^{\prime}, \vec{r}_{2}^{\prime}\right)
$$

Here $n_{i}$ and $\phi_{i}$ are its eigenvalues and eigenfunctions. For a spinless Fermi gas, the eigenfunction $\phi_{i}$ is odd under exchanges of two fermions $\phi_{i}\left(\vec{r}_{1}, \vec{r}_{2}\right)=-\phi_{i}\left(\vec{r}_{2}, \vec{r}_{1}\right)$. When two particles come very close to each other, the eigenfunction can be expanded using a two-body wave function (using translational invariance),

$$
\phi_{\vec{P}, m= \pm 1}\left(\vec{r}_{1}, \vec{r}_{2}\right)=\frac{e^{i \vec{P} \cdot \vec{R}} e^{i m \theta}}{\sqrt{2 \pi V}} \sum_{k} a_{\vec{P}, m, k} R_{k}(r) .
$$

Here $\vec{P}=\vec{p}_{1}+\vec{p}_{2}$ and $\vec{R}=\left(\vec{r}_{1}+\vec{r}_{2}\right) / 2$ are the center-of-mass momentum and position. $\theta$ describes the angle of relative coordinates $\vec{r}=\vec{r}_{1}-\vec{r}_{2} . a_{\vec{p}, m, k}$ is the expansion coefficient introduced such that (1) $\int d \vec{r}_{1} d \vec{r}_{2}\left|\phi\left(\vec{r}_{1}, \vec{r}_{2}\right)\right|=1$ is normalized and (2) the radial wave function $R_{k}(r)$ in the asymptotic regime $r_{0} \ll r \ll 1 / k$ is given by Eqs. (17) and (18). As a result, we have

$$
\begin{aligned}
\rho_{2}\left(\vec{r}_{1}, \vec{r}_{2} ; \vec{r}_{2}^{\prime}, \vec{r}_{1}^{\prime}\right)= & \sum_{\vec{P}, m} n_{\vec{P}, m} \frac{e^{i \vec{P} \cdot\left(\vec{R}-\vec{R}^{\prime}\right)} e^{i m\left(\theta-\theta^{\prime}\right)}}{2 \pi V} \\
& \times \sum_{k, k^{\prime}} a_{\vec{P}, m, k}^{*} a_{\vec{P}, m, k^{\prime}} R_{k}(r) R_{k^{\prime}}\left(r^{\prime}\right) .
\end{aligned}
$$

Here $r^{\prime}$ and $\theta^{\prime}$ are defined accordingly. As in Ref. [17], we have included possible bound states in the sum over $k$. The interaction energy can be expressed as [short-range potential $U(r)]$

$$
\begin{aligned}
\langle U\rangle & =\frac{1}{2} \int d^{2} r_{1} d^{2} r_{2} U\left(r_{1}-r_{2}\right) \rho_{2}\left(r_{1}, r_{2} ; r_{2}, r_{1}\right) \\
& =C_{a} \int d r r U(r) u_{0}^{2}(r)+2 C_{R} \int d r r U(r) u_{0}(r) u_{1}(r),
\end{aligned}
$$

where we have defined two $p$-wave contacts $C_{a}$ and $C_{R}$ as

$$
\begin{gathered}
C_{a} \equiv \frac{1}{2} \sum_{P, m, k^{\prime} k} n_{P, m} a_{P, m, k^{\prime}}^{*} a_{P, m, k}, \\
C_{R} \equiv \frac{1}{4} \sum_{P, m, k^{\prime} k} n_{P, m} a_{P, m, k^{\prime}}^{*} a_{P, m, k}\left(k^{\prime 2}+k^{2}\right) .
\end{gathered}
$$

By definition, $C_{a} \geqslant 0$, while $C_{R}$ has no definite signs. The variations of free energy $F$ as the interatomic potential is varied by $\delta U$ are given by

$$
\begin{aligned}
\delta F & =\delta\langle U\rangle \\
& =C_{a} \int d r r \delta U u_{0}^{2}(r)+2 C_{R} \int d r r \delta U u_{0}(r) u_{1}(r) .
\end{aligned}
$$

Using Eqs. (20) and (21), we obtain two adiabatic relations,

$$
\begin{gathered}
\frac{\partial F}{\partial a^{-1}}=-\frac{\pi}{2} C_{a}, \\
\frac{\partial F}{\partial \ln R}=C_{R} .
\end{gathered}
$$

Similar to the cases of $s$ - and $p$-wave scattering in three dimensions, the above two equations give important relationships between the two contacts $\left(C_{a}, C_{R}\right)$ and the thermodynamics of the system. Here we should mention that for a $p$-wave resonant Fermi gas in two dimensions, the effective potential resulting from the three-body correlation could support the so-called super Efimov states, for which the energy for three bodies has 
a double-exponential scaling law [31-33]. Very recently, the three-body contact arising from the super Efimov states was discussed in Ref. [34]. However, in the present paper, we shall focus on contacts arising from two-body correlations.

\section{UNIVERSAL RELATIONS}

The utility of contacts lies in the fact that they relate various physical observables and provide a crucial consistency check on theory and experiments.

\section{A. Tails of momentum distribution}

The derivation of momentum distribution follows that of Ref. [17]. The momentum distribution $n_{\vec{q}}$ is related to singleparticle density matrix $\rho_{1}\left(r, r^{\prime}\right)$,

$$
\rho_{1}\left(\vec{r}, \vec{r}^{\prime}\right) \equiv\left\langle\psi^{\dagger}(\vec{r}) \psi\left(\vec{r}^{\prime}\right)\right\rangle=\frac{1}{V} \sum_{\vec{q}} e^{i \vec{q} \cdot \vec{\rho}} n_{\vec{q}},
$$

where $\vec{\rho}=\vec{r}^{\prime}-\vec{r}$ and we have used the translational invariance of the system. In terms of a many-body wave function $\Psi\left(\vec{r}_{1}, \vec{r}_{2}, \ldots, \vec{r}_{N}\right)$, the single-particle density matrix can be written as

$$
\begin{aligned}
\rho_{1}\left(\vec{r}_{1}, \vec{r}_{1}^{\prime}\right)= & N \int d \vec{r}_{2} \cdots d \vec{r}_{N} \Psi^{*}\left(\vec{r}_{1}, \vec{r}_{2}, \ldots, \vec{r}_{N}\right) \\
& \times \Psi\left(\vec{r}_{1}^{\prime}, \vec{r}_{2}, \ldots, \vec{r}_{N}\right) .
\end{aligned}
$$

As a result,

$$
\begin{aligned}
n_{\vec{q}}= & \frac{1}{V} \int d^{2} \vec{r} d \vec{r}^{\prime} \rho_{1}\left(\vec{r}, \vec{r}^{\prime}\right) e^{-i \vec{q} \cdot\left(\vec{r}-\vec{r}^{\prime}\right)} \\
= & \frac{N}{V} \int d^{2} \vec{r} d \vec{r}^{\prime} d \vec{r}_{2} \ldots d \vec{r}_{N} e^{-i \vec{q} \cdot\left(\vec{r}-\vec{r}^{\prime}\right)} \Psi^{*}\left(\vec{r}, \vec{r}_{2}, \ldots, \vec{r}_{N}\right) \\
& \times \Psi\left(\vec{r}^{\prime}, \vec{r}_{2}, \ldots, \vec{r}_{N}\right) .
\end{aligned}
$$

Now, we need $\vec{r}$ to be close to $\vec{r}^{\prime}$ in order to extract the highmomentum distribution. This requires one of the coordinates $\vec{r}_{2} \cdots \vec{r}_{N}$ to be close to both $\vec{r}$ and $\vec{r}^{\prime}$, which gives the singular contribution to the one-body density matrix. Note that in total there are $N-1$ possibilities, and we finally obtain $n_{\vec{q}}$ in terms of the singular part of the two-body density matrix,

$$
\begin{aligned}
n_{\vec{q}}= & \frac{1}{V^{2}} \sum_{\vec{P} m k^{\prime} k} n_{\vec{P}, m} a_{\vec{P}, m, k^{\prime}}^{*} a_{\vec{P}, m, k} \int d^{2} \vec{r} d^{2} \vec{r}^{\prime} e^{i(\vec{P} / 2-\vec{q}) \cdot\left(\vec{r}-\vec{r}^{\prime}\right)} \\
& \times\left\{\int d^{2} \vec{r}_{1} \frac{e^{i m \theta}}{\left|\vec{r}-\vec{r}_{1}\right|} \frac{e^{-i m \theta^{\prime}}}{\left|\vec{r}^{\prime}-\vec{r}_{1}\right|}-\left(k^{\prime 2}+k^{2}\right) \int d^{2} \vec{r}_{1}\right. \\
& \left.\times \frac{e^{i \theta}\left|\vec{r}-\vec{r}_{1}\right|}{2} \ln \frac{\left|\vec{r}-\vec{r}_{1}\right|}{\tilde{R}} \frac{e^{-i \theta^{\prime}}}{\left|\vec{r}^{\prime}-\vec{r}_{1}\right|}\right\} \\
= & \frac{(2 \pi)^{2}}{V} \sum_{\vec{P} m k^{\prime} k} n_{\vec{P}, m} a_{\vec{P}, m, k^{\prime}}^{*} a_{\vec{P}, m, k} \\
& \times\left\{\frac{1}{q^{2}}+\frac{\left(k^{\prime 2}+k^{2}\right)+\vec{P} \cdot \vec{q}-P^{2} / 4}{q^{4}}\right\} .
\end{aligned}
$$

That is,

$$
n_{\vec{q}} \sim \frac{4 \pi}{V}\left\{\frac{C_{a}}{q^{2}}+\frac{2 C_{R}-C_{P}}{q^{4}}\right\}
$$

where

$$
C_{P} \equiv \frac{\pi}{4} \sum_{P, m, k^{\prime} k} n_{P, m} a_{P, m, k^{\prime}}^{*} a_{P, m, k} P^{2},
$$

which arises from the center-of-mass motion of the pairs. In the above derivation, we have assumed that the system respects inversion symmetry so that the linear term in $\vec{P}$ vanishes.

\section{B. Pressure relation and virial theorem}

To derive the pressure relation and the virial theorem, it is enough to invoke the dimensional analysis. The free energy $F$ of the system should be of the form $F=$ $N \epsilon_{F} f\left(T / T_{F}, k_{F}^{2} a, k_{F} R\right)$, where $f$ is a dimensionless function, $\epsilon_{F}=T_{F} \equiv k_{F}^{2} / 2=2 \pi n$ is Fermi energy $\left(k_{B}=1\right), k_{F}$ is Fermi momentum, and $n$ is particle density. From the thermodynamic relation,

$$
\begin{aligned}
p & =-\frac{\partial F}{\partial V}=-\frac{\partial F}{\partial k_{\mathrm{F}}} \frac{\partial k_{\mathrm{F}}}{\partial V} \\
& =\frac{E}{V}+\frac{1}{V} \frac{\pi C_{a}}{2 a}+\frac{C_{R}}{2 V} .
\end{aligned}
$$

In the last line we have used the adiabatic theorems. Similarly, one can extend the virial theorem in a harmonic trap $V_{\text {trap }}(r)=$ $\frac{1}{2} M \omega^{2} r^{2}$; then the total energy can be written as

$$
E \equiv E(N, \omega, a, R)=\hbar \omega \varepsilon\left(N, a / \ell^{2}, R / \ell\right),
$$

where $\varepsilon$ is a dimensionless function and $\ell=\sqrt{\hbar / M \omega}$ is the oscillator length. Taking the derivative with respect to the oscillator frequency $\omega$, we have

$$
\frac{\partial E}{\partial \omega}=\frac{2\left\langle V_{\text {trap }}\right\rangle}{\omega} .
$$

Carrying out manipulations similar to those in the uniform case, we find

$$
2\left\langle V_{\text {trap }}\right\rangle=E+\frac{\pi}{2} \frac{C_{a}}{a}+\frac{C_{R}}{2} .
$$

In both Eqs. (41) and (44), when $a \rightarrow \pm \infty$ (at resonance), the second contact $C_{R}$ breaks the scaling invariance of the two-dimensional $p$-wave Fermi gas at resonance.

\section{EXPLICIT CALCULATIONS}

To gain more insight into the behavior of $p$-wave contacts defined by the adiabatic theorem, we discuss two explicit calculations of them in the following.

\section{A. Two-body bound state}

Let us choose a cutoff scale $r_{c}$ as before and write down the wave function for $r \geqslant r_{c}\left(r_{c} \gg r_{0}\right)$ in the center-of-mass coordinates as

$$
\psi^{>}(r)=\alpha K_{1}(\kappa r)
$$

where $\alpha$ is a normalization constant and $K_{1}(r)$ is the modified Bessel function of the second kind. The bound-state energy $E_{b}=-\kappa^{2}$. For $0 \leqslant r \leqslant r_{c}$, the wave function is

$$
\psi^{<}(r)=\alpha \frac{K_{1}\left(\kappa r_{c}\right)}{u_{0}\left(r_{c}\right)} u_{0}(r) .
$$


The normalization constant $\alpha$ is determined by, near resonance $1 / a \sim 0$,

$$
\begin{aligned}
\frac{1}{\alpha^{2}}= & 2 \pi\left\{\int_{0}^{r_{c}} d r r\left|\psi^{<}(r)\right|^{2}+\int_{r_{c}}^{\infty} d r r\left|\psi^{>}(r)\right|^{2}\right\} \\
= & \frac{2 \pi}{\kappa^{2} r_{c}^{2}} \frac{1}{\left(-\frac{1}{r_{c}}+\frac{\pi r_{c}}{4 a}\right)^{2}}\left[\ln \frac{e^{\gamma_{E}} r_{c} / 2}{R}-\frac{\pi r_{c}^{2}}{4 a}+\frac{\pi^{2} r_{c}^{4}}{64 a^{2}}\right] \\
& +\frac{2 \pi}{\kappa^{2}}\left[-1 / 2-\gamma_{E}+\ln 2-\ln \left(\kappa r_{c}\right)+O\left(\left(\kappa r_{c}\right)^{2}\right)\right] \\
\simeq & -\frac{2 \pi[\ln (\kappa R)+1 / 2]}{\kappa^{2}} .
\end{aligned}
$$

Here we have used Bethe's integral formula. Expanding the wave function when $\kappa r \ll 1$,

$$
\begin{aligned}
\left|\psi^{>}(r)\right|^{2} & =\alpha^{2}\left|K_{1}(\kappa r)\right|^{2} \\
& \simeq \alpha^{2}\left(\frac{1}{\kappa^{2} r^{2}}+\ln r+\cdots\right) \\
& =-\frac{\kappa^{2}}{2 \pi[\ln (\kappa R)+1 / 2]}\left(\frac{1}{\kappa^{2} r^{2}}+\ln r+\cdots\right)
\end{aligned}
$$

at low energy, $\kappa R \ll 1$. We can now read off the contacts from the above equation:

$$
\begin{gathered}
C_{a}=\frac{-1}{\ln (\kappa R)+1 / 2}>0, \\
C_{R}=\frac{\kappa^{2}}{\ln (\kappa R)+1 / 2}<0 .
\end{gathered}
$$

Using the equation for the bound-state energy (14), one can verify the adiabatic theorems, namely, that $\frac{\partial E_{b}}{\partial a^{-1}}=-\frac{\pi}{2} C_{a}$ and $\frac{\partial E_{b}}{\partial \ln R}=C_{R}$.

\section{B. Virial expansion}

In this section, we calculate the contact parameters at high temperature by virial expansion $[35,36]$. The pressure $p$ and the inverse volume (area) $1 / v$ can be expanded in terms of powers of fugacity $z=e^{\mu / T}$ [37]:

$$
\begin{gathered}
\frac{p}{T}=\frac{1}{\lambda^{2}} \sum_{l} b_{l} z^{l}, \\
\frac{1}{v}=N / V=\frac{1}{\lambda^{2}} \sum_{l} l b_{l} z^{l} .
\end{gathered}
$$

Here $T$ is temperature, $\lambda=\sqrt{2 \pi / T}\left(k_{B}=\hbar=1\right)$ is the de Broglie wavelength, and $\mu$ is the chemical potential. The grand potential is

$$
\Omega=-p V=-T V \frac{1}{\lambda^{2}} \sum_{l} b_{l} z^{l} .
$$

The equation of state can be obtained by eliminating the fugacity $z$

$$
\frac{p v}{T}=\sum_{l=1}^{\infty} a_{l}(T)\left(\frac{\lambda^{2}}{v}\right)^{l-1},
$$

where $a_{l}$ is virial coefficient. For example,

$$
\begin{aligned}
& a_{1}=b_{1}=1, \quad a_{2}=-b_{2}, \quad a_{3}=4 b_{2}^{2}-2 b_{3}, \\
& a_{4}=-20 b_{2}^{3}+18 b_{2} b_{3}-3 b_{4} .
\end{aligned}
$$

For a 2D ideal Fermi gas, the inverse area $\frac{1}{v}=$ $N / V=\sum_{k} \frac{z e^{-\beta k^{2} / 2}}{1+z e^{-\beta k^{2} / 2}}=\frac{\ln (1+z)}{\lambda^{2}}=\frac{1}{\lambda^{2}} \sum_{n}(-1)^{n-1} \frac{z^{n}}{n}$, so $b_{l}^{0}=$ $(-1)^{l-1} / l^{2}$. Including the interaction effects, the correction to the second virial coefficient is given by

$$
\Delta b_{2}=4\left\{\sum_{b} e^{-E_{b} / T}+\frac{1}{\pi} \int_{0}^{\infty} d k \frac{\partial \delta_{1}}{\partial k} e^{-k^{2} / T}\right\} .
$$

The summation on the right-hand side includes the effects of possible bound states, while the integral takes into account the scattering fermions. The phase shift $\delta_{1}$ was given before explicitly,

$$
\frac{\partial \delta_{1}(k)}{\partial k}=-\frac{2\left(\frac{1}{a k^{3}}+\frac{1}{\pi k}\right)}{1+\left(-\frac{1}{a k^{2}}+\frac{2 \ln (R k)}{\pi}\right)^{2}} .
$$

The contacts can be obtained from the thermodynamics potential $\Omega$,

$$
\begin{gathered}
\frac{\partial \Omega}{\partial\left(a^{-1}\right)}=-\frac{\pi}{2} C_{a}=-T V \frac{z^{2}}{\lambda^{2}} \frac{\partial \Delta b_{2}}{\partial a^{-1}}, \\
\frac{\partial \Omega}{\partial(\ln R)}=C_{R}=-T V \frac{z^{2}}{\lambda^{2}} \frac{\partial \Delta b_{2}}{\partial \ln R},
\end{gathered}
$$

where $z$ can be obtained approximately from the equation of state for a classical ideal gas, e.g., $z=e^{n \lambda^{2}}-1 \simeq n \lambda^{2}$. So one finally obtains

$$
\begin{aligned}
& C_{a}=\frac{2}{\pi} T V n^{2} \lambda^{2} \frac{\partial \Delta b_{2}}{\partial a^{-1}}=\frac{2}{\pi} 2 \pi N n \frac{\partial \Delta b_{2}}{\partial a^{-1}}, \\
& C_{R}=-T V n^{2} \lambda^{2} \frac{\partial \Delta b_{2}}{\partial \ln R}=-2 \pi N n \frac{\partial \Delta b_{2}}{\partial \ln R},
\end{aligned}
$$

where $N$ is the particle number and $n$ is particle density. Figure 2 shows how the second virial coefficient $\Delta b_{2}$ and the contacts $C_{a}$ and $C_{R}$ change as a function of interaction parameters $1 / a$ at fixed $R$. Because $C_{a} \geqslant 0, \Delta b_{2}$ is monotonically increasing with $a^{-1}$. Generally speaking, close to resonance
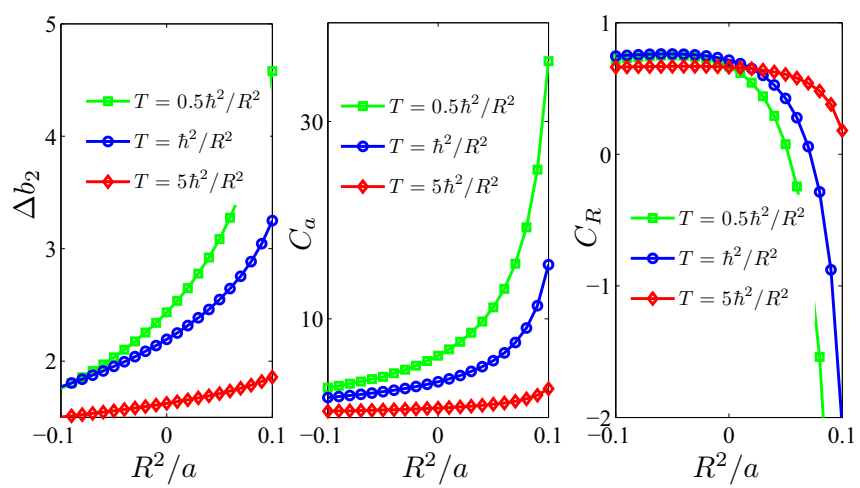

FIG. 2. The variations of contacts $C_{a}$ and $C_{R}$ with the interaction parameter $1 / a$ for different temperatures. Here we use the effective range $R$ as the fundamental length scale, and the units of $C_{a}$ and $C_{R}$ as plotted are $2 \pi N n R^{2} \hbar^{2} / M$ and $2 \pi N n \hbar^{2} / M$, respectively. 
$1 / a \sim 0$, the interaction effects $\Delta b_{2}$ diminish with increasing temperature. Because the contribution of bound states to the contact $C_{R}$ is negative, with increasing attractive interaction $\left(1 / a \rightarrow 0_{+}\right)$, the contact $C_{R}$ decreases and eventually becomes negative.

\section{BREATHING MODE AND CONTACTS}

It is known that for scaling-invariant interactions in two dimensions, $U(\lambda \rho)=U(\rho) / \lambda^{2}$, where $\lambda$ is an arbitrary scaling factor, the system has $\mathrm{SO}(2,1)$ symmetry, and the frequency of the breathing mode in the harmonic trap is exactly twice the trap frequency $2 \omega$ [24]. This applies, for example, for the cases of delta contact $\delta^{2}\left(\vec{r}_{1}-\vec{r}_{2}\right)$ or the inverse-square potential $1 /\left|\vec{r}_{1}-\vec{r}_{2}\right|^{2}$. However, true interatomic interactions break the scaling invariance, and this leads to the frequency shift of breathing modes. In a two-component Fermi gas with $s$-wave interactions, one finds that this shift is related to the contact of the system [38,39], and in particular, at unitarity, the scaling invariance is regained [40]. However, quite differently from a single-component Fermi gas with $p$-wave interaction in two dimensions, the scaling invariance is also broken even at resonance when $a \rightarrow \infty$ due to the existence of the contact $C_{R}$. In the following, we calculate the breathing-mode frequency shift and relate it to the two $p$-wave contacts $C_{a}$ and $C_{R}$.

The Hamiltonian in a trapped system is given by $H=$ $H_{0}+V_{\text {trap }}+H_{\text {int }}$, where $H_{0}=\frac{1}{2} \sum_{i} p_{i}^{2}$ is the kinetic energy (recall $M=\hbar=1$ ). We consider the isotropic harmonic trap in two dimensions,

$$
V_{\text {trap }}=\frac{\omega^{2}}{2} \sum_{i} r_{i}^{2}
$$

where $\omega$ is the trapping frequency. The interaction between the atoms $H_{\text {int }}$ is of the standard form $H_{\text {int }}=\sum_{i<j} U\left(\vec{r}_{i}-\vec{r}_{j}\right)$. The excited operator of the breathing modes

$$
O \equiv \sum_{i} r_{i}^{2}=\frac{2}{\omega^{2}} V_{\text {trap }}
$$

The equation of motion of operator $O$

$$
i \frac{\partial O(t)}{\partial t}=[O, H]=\sum_{i}\left[\vec{r}_{i}^{2}, \vec{p}_{i}^{2} / 2\right]=2 i D .
$$

Here $D \equiv \sum_{i}\left[\vec{r}_{i} \cdot \vec{p}_{i}+\vec{p}_{i} \cdot \vec{r}_{i}\right] / 2$ is the dilation operator. The equation of motion of $D$ is given by

$$
i \frac{\partial D}{\partial t}=[D, H]=\left[D, H_{0}+V_{\text {trap }}+H_{\text {int }}\right] .
$$

The various commutators can be evaluated explicitly. In particular, we have

$$
\begin{gathered}
{\left[D, H_{0}\right]=2 i H_{0},} \\
{\left[D, V_{\text {trap }}\right]=-2 i V_{\text {trap }},}
\end{gathered}
$$

and

$$
\left[D, H_{\mathrm{int}}\right]=-i \sum_{i<j}\left[\vec{r}_{i j} \cdot \frac{\partial}{\partial \vec{r}_{i j}}\right] U\left(\vec{r}_{i}-\vec{r}_{j}\right),
$$

where $\vec{r}_{i j}=\vec{r}_{i}-\vec{r}_{j}$. As a result, one finds that

$$
\begin{aligned}
\frac{\partial^{2} O(t)}{\partial t^{2}} & =2 \frac{\partial D}{\partial t} \\
& =4 H_{0}-4 V_{\text {trap }}-2 \sum_{i<j}\left[\vec{r}_{i j} \cdot \frac{\partial}{\partial \vec{r}_{i j}}\right] U\left(\vec{r}_{i}-\vec{r}_{j}\right) .
\end{aligned}
$$

Using the fact that $\hat{\rho}_{2}(\vec{r}, t) \equiv 2 \sum_{i<j} \delta^{2}\left[\vec{r}(t)-\vec{r}_{i j}\right]$, we can write down the equation of motion for the average value of $O$ as

$$
\begin{aligned}
\frac{\partial^{2}\langle O(t)\rangle}{\partial t^{2}}= & 4\langle H\rangle-4 \omega^{2}\langle O\rangle \\
& -\int d^{2} \vec{r}\left[2 U(r)+\vec{r} \cdot \frac{\partial U(r)}{\partial \vec{r}}\right] \rho_{2}(r, t) .
\end{aligned}
$$

In the case of a scaling-invariant potential in two dimensions, $2 U(r)+\vec{r} \cdot \frac{\partial U(r)}{\partial \vec{r}} \equiv 0$, the interaction effects on the breathingmode frequency shift vanish exactly. In the Appendix A, we show that the interaction correction can be written in terms of $p$-wave contacts,

$$
\int d^{2} \vec{r}\left[2 U(r)+\vec{r} \cdot \frac{\partial U(r)}{\partial \vec{r}}\right]\left\langle\hat{\rho}_{2}(r, t)\right\rangle=-\frac{2 \pi C_{a}(t)}{a}-2 C_{R}(t) .
$$

As a result, the equation of motion for the breathing-mode operator $O$ is given by, when taking the expectation value of the many-body state,

$$
\frac{\partial^{2}\langle O(t)\rangle}{\partial t^{2}}=4 E-4 \omega^{2}\langle O(t)\rangle+\frac{2 \pi C_{a}(t)}{a}+2 C_{R}(t) .
$$

The time dependences of the contacts give rise to the correction of the breathing-mode frequency. We note that the average energy $E \equiv\langle H\rangle$ does not depend on time $t$ because of $[H, H]=0$. In a stationary state, the virial theorem is recovered:

$$
2 V_{\text {trap }}=E+\frac{\pi}{2 a} C_{a}+\frac{C_{R}}{2} .
$$

When the $p$-wave contacts are zero, the scaling invariance is restored, and the frequency of the breathing mode is exactly $2 \omega$. Near the resonance $1 / a \rightarrow 0$, although the $C_{a}$ term vanishes, $C_{R}$ is still finite, and this breaks the scaling invariance even at resonance.

In the following, we will investigate the breathing-mode frequency shift in the high-temperature limit. We write the density distribution in the trap during the breathing motion of the cloud in the following scaling form:

$$
n(\vec{r}, t)=\frac{1}{\gamma^{2}(t)} n_{0}\left(\frac{\vec{r}}{\gamma(t)}\right),
$$

where $\gamma(t)=1+\Delta \gamma(t), \Delta \gamma(t) \ll 1$ for small oscillations. Here $n_{0}(\vec{r})$ is the density distribution of the equilibrium, and the particle number $N=\int d^{2} \vec{r} n_{0}(\vec{r})$. On the other hand, 
the expectation value of the breathing-mode operator can be written as

$$
\begin{aligned}
\langle O(t)\rangle & =\int d^{2} \vec{r} r^{2} n(\vec{r}, t)=\gamma^{2}(t) \int d^{2} \vec{r} r^{2} n_{0}(\vec{r}) \\
& \simeq[1+2 \Delta \gamma]\langle O\rangle,
\end{aligned}
$$

where $\langle O\rangle \equiv \int d^{2} \vec{r} r^{2} n_{0}(\vec{r})$ is the average value of $O$ in equilibrium. Similarly, from the high-temperature virial expansion, Eqs. (62) and (63), and using the local-density approximation, we get

$$
\begin{aligned}
C_{a}(t) & =\frac{\left\langle C_{a}\right\rangle}{\gamma^{2}}, \\
C_{R}(t) & =\frac{\left\langle C_{R}\right\rangle}{\gamma^{2}} .
\end{aligned}
$$

Here $\quad\left\langle C_{a}\right\rangle \equiv \frac{2}{\pi} T \lambda^{2} \frac{\partial \Delta b_{2}}{\partial a^{-1}} \int d^{2} \vec{r} n_{0}^{2}(\vec{r}) \quad$ and $\quad\left\langle C_{R}\right\rangle \equiv$ $-T \lambda^{2} \frac{\partial \Delta b_{2}}{\partial \ln R} \int d^{2} \vec{r} n_{0}^{2}(\vec{r})$ are contact values in equilibrium.

$$
\frac{\partial^{2} \Delta \gamma(t)}{\partial t^{2}}+\left[4 \omega^{2}+\frac{2 \pi\left\langle C_{a}\right\rangle / a+2\left\langle C_{R}\right\rangle}{\langle O\rangle}\right] \Delta \gamma(t)=0 .
$$

One thus finds that for a small breathing motion of the cloud, the frequency is given by

$$
\omega_{\mathrm{b}}=\sqrt{4 \omega^{2}+\frac{2 \pi\left\langle C_{a}\right\rangle / a+2\left\langle C_{R}\right\rangle}{\langle O\rangle}} .
$$

Assuming that the shift is small, one can expand $\omega_{\mathrm{b}}$ and find

$$
\Delta \omega \equiv \omega_{\mathrm{b}}-2 \omega=\frac{1}{2 \omega\langle O\rangle}\left[\frac{\pi\left\langle C_{a}\right\rangle}{a}+\left\langle C_{R}\right\rangle\right] .
$$

One can evaluate the frequency shift at high temperature, where the density distribution can be approximated by the classic Boltzmann distribution $n_{0}(\vec{r})=A \exp \left(-\omega^{2} \vec{r}^{2} / 2 T\right)$, where $A=N \omega^{2} /(2 \pi T)$ and $N$ is the particle number. So the frequency shift becomes

$$
\Delta \omega=\frac{N \omega^{3}}{8 T^{2}}\left[\frac{2}{a} \frac{\partial \Delta b_{2}}{\partial a^{-1}}-\frac{\partial \Delta b_{2}}{\partial \ln R}\right] .
$$

Near the resonance $1 / a \rightarrow 0$, the frequency shift arises only from the contact of effective range $C_{R}$. Note that unlike the $p$-wave contacts for three dimensions, $C_{R}$ does not vanish at resonance. Here we take $T=2 E_{F}$ with the Fermi energy related to the radial trap frequency $E_{F}=\hbar \omega \sqrt{2 N}$ in two dimensions, and the breathing-mode frequency shift $\Delta \omega$ at high temperature $T=0.1 / R^{2}$ is given in Fig. 3 . Note that it is finite at resonance when $1 / a=0$ and can be negative on the BCS side of the resonance when $1 / a<0$. On the Bose-Einstein condensate side of the resonance $1 / a>0$, the frequency shift is always positive and becomes larger and larger as the attractive interaction becomes strong $(a \rightarrow 0+)$.

\section{SUMMARY}

In this paper, we have extended the concepts of $p$-wave contacts to two dimensions and have derived the related universal relations. In particular, we have shown how the $p$ wave contacts change the breathing-mode frequency from the

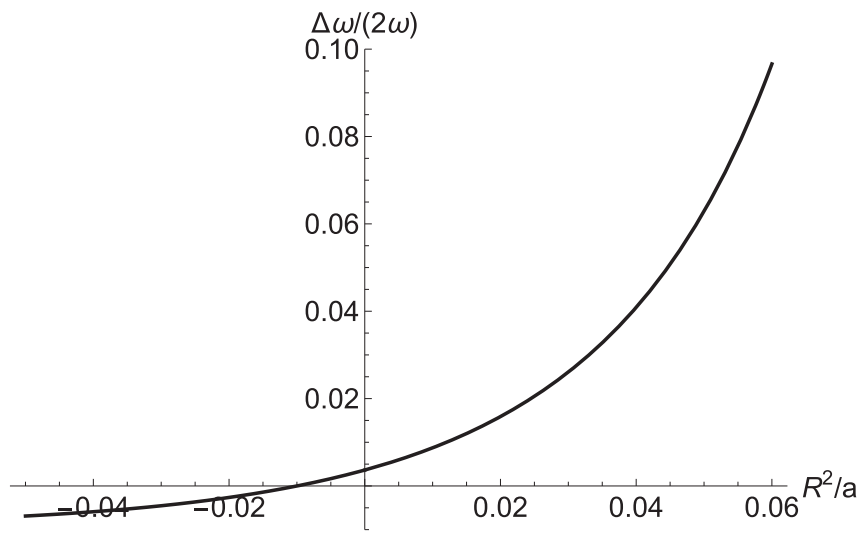

FIG. 3. The frequency shift $\Delta \omega /(2 \omega)$ of breathing modes as a function of $R^{2} / a$ near the resonance.

scaling-invariant result. Specific results were obtained in the high-temperature limit where the $2 \mathrm{D}$ system is more stable. It would be interesting if further experiments in two dimensions could be conducted, and in the Appendix B, we consider realistic trap parameters and show how the effective twodimensional scattering parameters depend on the magnetic field.

\section{ACKNOWLEDGMENTS}

We would like to thank Z. Yu and J. H. Thywissen for useful discussions. This research was supported by the Hong Kong Research Grants Council, General Research Fund, HKU 17306414, 17318316 and Collaborative Research Fund, HKUST3/CRF/13G and the Croucher Foundation under the Croucher Innovation Award.

\section{APPENDIX A：DERIVATION OF EQUATION (74)}

According to the definition of the $p$-wave contacts, the two-body density matrix $\rho_{2}\left(\vec{r}_{1}, \vec{r}_{2} ; \vec{r}_{2}, \vec{r}_{1}\right)\left[\equiv \rho_{2}\left(\vec{r}_{1}-\vec{r}_{2}\right)\right]$ in the short range can be written as

$$
\rho_{2}(r)=\frac{C_{a}}{\pi} u_{0}^{2}(r)+\frac{2 C_{R}}{\pi} u_{0}(r) u_{1}(r),
$$

where $r=\left|\vec{r}_{1}-\vec{r}_{2}\right|$. This gives the possibility of finding two two-particle distance $r$ apart. So Eq. (73) can be written as

$$
\frac{\partial^{2}\langle O(t)\rangle}{\partial t^{2}}=4\langle H\rangle-4 \omega^{2}\langle O(t)\rangle-(A+B),
$$

where

$$
\begin{array}{r}
A=2 C_{a} \int d r r\left[2 U(r)+r \frac{\partial U(r)}{\partial r}\right] u_{0}^{2}(r), \\
B=2 C_{R} \int d r r\left[2 U(r)+r \frac{\partial U(r)}{\partial r}\right] 2 u_{0}(r) u_{1}(r) .
\end{array}
$$

Our task below will be to evaluate the above two expressions. One useful fact to notice is that since $U(r)$ is a short-range function, the integrals are effectively cut off at $r_{c}$ because $U(r)=0$ when $r \geqslant r_{c}$. As a result, we can write $A$, and 
integrating from zero to $r_{c}$, we find

$$
\begin{aligned}
A & =2 C_{a} \int d r r\left[2 U(r)+r \frac{\partial U(r)}{\partial r}\right] u_{0}^{2}(r) \\
& =-4 C_{a} \int d r r^{2} U(r) u_{0}(r) \frac{\partial u_{0}(r)}{\partial r} .
\end{aligned}
$$

In the above derivation, we have integrated the second term by parts and used the fact that $\left.U(r) r^{2} u_{0}^{2}(r)\right|_{0} ^{r_{c}}=0$. Using a differential equation for $u_{0}(r)$,

$$
\left[\frac{1}{r} \frac{\partial}{\partial r}\left(r \frac{\partial}{\partial r}\right)-\frac{1}{r^{2}}\right] u_{0}(r)=U(r) u_{0}(r),
$$

and multiplying both sides by $r^{2} u_{0}^{\prime}(r)$ and integrating from zero to $r_{c}$, we find, using $u_{0}(r=0)=0,\left.r \frac{\partial u_{0}(r)}{\partial r}\right|_{r=0}=0$, and Eq. (17),

$$
\begin{aligned}
A & =-2 C_{a}\left[\left(r \frac{\partial u_{0}(r)}{\partial r}\right)^{2}-u_{0}^{2}(r)\right]_{0}^{r_{c}} \\
& =-2 C_{a}\left[\left(\frac{1}{r_{c}}+\frac{\pi r_{c}}{4 a}\right)^{2}-\left(-\frac{1}{r_{c}}+\frac{\pi r_{c}}{4 a}\right)^{2}\right] \\
& =-2 \pi \frac{C_{a}}{a} .
\end{aligned}
$$

The calculation of $B$ needs more effort. Let us first consider the following integral:

$$
2 C_{R} \int d r r\left[2 U(r)+r \frac{\partial U(r)}{\partial r}\right] R_{k}^{2}(r),
$$

where $R_{k}(r)=u_{0}(r)+k^{2} u_{1}(r)$ is the radial wave function expanded to second order in $k$. $B$ is simply given by the coefficient of $k^{2}$ in the above integral. $R_{k}(r)$ obeys the following differential equation:

$$
\left[\frac{1}{r} \frac{\partial}{\partial r}\left(r \frac{\partial}{\partial r}\right)-\frac{1}{r^{2}}\right] R_{k}(r)+k^{2} R_{k}(r)=U(r) R_{k}(r) .
$$

Multiply both sides by $r^{2} R_{k}^{\prime}(r)$ and integrating it by parts, one finds

$$
\begin{aligned}
& \int_{0}^{r_{c}} U(r) r^{2} R_{k}^{\prime}(r) R_{k}(r) d r \\
& \quad=k^{2} \int_{0}^{r_{c}} r^{2} R_{k}^{\prime}(r) R_{k}(r) d r+\frac{1}{2}\left[\left(r \frac{\partial R_{k}(r)}{\partial r}\right)^{2}-R_{k}^{2}(r)\right]_{0}^{r_{c}} .
\end{aligned}
$$

Like for $A$, we have

$$
\begin{aligned}
& \int d r r\left[2 U(r)+r \frac{\partial U(r)}{\partial r}\right] R_{k}^{2}(r) \\
& =-2 \int d r r^{2} U(r) R_{k}(r) \frac{\partial R_{k}(r)}{\partial r} \\
& =-2 k^{2} \int_{0}^{r_{c}} r^{2} R_{k}^{\prime}(r) R_{k}(r) d r-\left[\left(r \frac{\partial R_{k}(r)}{\partial r}\right)^{2}-R_{k}^{2}(r)\right]_{0}^{r_{c}} .
\end{aligned}
$$

The coefficient of $k^{2}$ in the above expression determines $B$ (up to a factor $\left.2 C_{R}\right)$. Now, since $\left.\left\{\left(r \frac{\partial R_{k}(r)}{\partial r}\right)^{2} / 2-\left[R_{k}^{2}(r)\right]^{2} / 2\right\}\right|_{r=0}=$
0 and $R_{k}\left(r_{c}>r_{0}\right)=u_{0}\left(r_{c}\right)+k^{2} u_{1}\left(r_{c}\right)$, the coefficient of $k^{2}$ is given as

$$
-4 C_{R}\left[\frac{1}{2}-\frac{\pi^{2} r_{c}^{4}}{64 a^{2}}+\ln \frac{r_{c}}{\tilde{R}}+\int_{0}^{r_{c}} d r r^{2} u_{0}(r) u_{0}^{\prime}(r)\right]
$$

The integral $\int_{0}^{r_{c}} d r r^{2} u_{0}(r) u_{0}^{\prime}(r)$ can be calculated from Bethe's integral formula. We first transform the limit of integration $r_{c} \rightarrow \lambda r_{c}$

$$
\int_{0}^{\lambda r_{c}} r u_{0}^{2}(r) d r=\ln \frac{e^{\gamma_{E}} \lambda r_{c} / 2}{R}-\frac{\pi \lambda^{2} r_{c}^{2}}{4 a}+\frac{\pi^{2} \lambda^{4} r_{c}^{4}}{64 a^{2}} .
$$

Then we can change the integral variable $r=\lambda x$,

$$
\int_{0}^{r_{c}} x u_{0}^{2}(\lambda x) d x=\ln \frac{e^{\gamma_{E}} \lambda r_{c} / 2}{R} / \lambda^{2}-\frac{\pi r_{c}^{2}}{4 a}+\frac{\pi^{2} \lambda^{2} r_{c}^{4}}{64 a^{2}} .
$$

Taking the derivative with respect to $\lambda$ and then setting $\lambda=1$, we get

$$
\int_{0}^{r_{c}} x^{2} u_{0}(x) u_{0}^{\prime}(x) d x=-\ln \frac{e^{\gamma_{E}} r_{c} / 2}{R}+\frac{1}{2}+\frac{\pi^{2} r_{c}^{4}}{64 a^{2}} .
$$

Finally, we obtain

$$
A+B=-\frac{2 \pi}{a} C_{a}-2 C_{R}
$$

and

$$
\frac{\partial^{2}\langle O(t)\rangle}{\partial t^{2}}=4 E-4 \omega^{2}\langle O(t)\rangle+\frac{2 \pi}{a} C_{a}(t)+2 C_{R}(t) .
$$

\section{APPENDIX B: THE RELATION BETWEEN THE EFFECTIVE SCATTERING PARAMETERS ( $a$ AND $R$ ) IN TWO DIMENSIONS AND THE THREE-DIMENSIONAL $p$-WAVE SCATTERING PARAMETERS}

It is suggested that the $2 \mathrm{D}$ strongly interacting $p$-wave Fermi gas might be more stable than its three-dimensional (3D) counterpart [41]. The 2D fermion gas may be produced from 3D fermion gas by using strong harmonic confinement along the $\hat{z}$ direction $[42,43]$. Let us then consider two spinless fermions moving in a harmonic potential of the form

$$
V_{z}(x, y, z)=\frac{1}{2} M \omega_{z}^{2} z^{2} .
$$

Thus the potential energy of the two particles is $\frac{1}{2}(2 M) \omega_{z}^{2} Z^{2}+$ $\frac{1}{2} \frac{M}{2} z^{2}$, where $2 Z=z_{1}+z_{2}$ is twice the center-of-mass $z$ coordinate and $z=z_{1}-z_{2}$ gives the relative coordinates. Since the center-of-mass motion is separated in the harmonic trap, one can write the Hamiltonian for the relative motion,

$$
H_{\mathrm{rel}}=\frac{1}{2 \mu}\left(p_{x}^{2}+p_{y}^{2}+p_{z}^{2}\right)+\frac{1}{2} \mu \omega_{z}^{2} z^{2},
$$

where $\mu=M / 2$ is the effective mass. In the following, we use units such that $M=1$, so $2 \mu=M=1$. We introduce $\vec{\rho} \equiv$ $x \hat{x}+y \hat{y}$ as the $x y$-plane projection of the relative coordinate $\vec{r}=\vec{r}_{1}-\vec{r}_{2}$ and $\rho=\sqrt{x^{2}+y^{2}}$. The free Green's function for 
the relative motion is given by

$$
G^{ \pm}\left(\vec{r}, \vec{r}^{\prime} ; E\right)=\left\langle\vec{r}_{1}\left|\frac{1}{E \pm i \eta-H_{\mathrm{rel}}}\right| \vec{r}_{2}\right\rangle .
$$

In the following, we shall shift the reference point of energy $\epsilon \equiv E-\omega_{z} / 2$. We are interested in the scattering of two fermions in the $x y$ plane, so let us set $z_{1}=z_{2}=0$ and introduce a complete set of harmonic oscillator states:

$$
G^{ \pm}(\rho) \equiv \frac{1}{V} \sum_{k, n} \frac{e^{i \vec{k} \cdot \vec{\rho}} \phi_{n}(0) \phi_{n}^{*}(0)}{\epsilon \pm i \eta-\left(k_{x}^{2}+k_{y}^{2}+n \omega_{z}\right)} .
$$

Here $\vec{k}=\left(k_{x}, k_{y}\right)$ lies in the $x y$ plane. $G^{ \pm}(\rho)$ describes the outgoing and incoming waves in two dimensions. Harmonic oscillator wave functions

$$
\phi_{n}(z)=\left[\frac{\alpha}{\sqrt{\pi 2^{n} n !}}\right]^{1 / 2} H_{n}(\alpha z),
$$

where $\alpha=\sqrt{\mu \omega_{z} / \hbar}=\sqrt{\omega_{z} / 2}$. The scattering wave function of the relative motion can then be written as

$$
\psi=\frac{\partial G^{-}(\rho)}{\partial \rho}-S \frac{\partial G^{+}(\rho)}{\partial \rho} .
$$

The stationary scattering wave function of the relative motion is a superposition of the incoming and the outgoing waves, the coefficient of which is the diagonalized $S$-matrix element $S=\exp \left(2 i \delta_{1}\right)$ in terms of the scattering phase shift $\delta_{1}$. In the following, we consider low-energy scattering when $0<\epsilon<$ $\omega_{z}$. To proceed further, we split the summation over $n$ into two parts, one with $n=0$ and another with $n>0, G^{ \pm}(\rho) \equiv$ $G_{1}^{ \pm}(\rho)+G_{2}^{ \pm}(\rho)$, with

$$
\begin{gathered}
G_{1}^{ \pm}(\rho) \equiv \frac{1}{V} \sum_{k} \frac{e^{i \vec{k} \cdot \vec{\rho}} \phi_{0}(0) \phi_{0}^{*}(0)}{\epsilon \pm i \eta-\left(k_{x}^{2}+k_{y}^{2}\right)}, \\
G_{2}^{ \pm}(\rho) \equiv \frac{1}{V} \sum_{k, n>0} \frac{e^{i \vec{k} \cdot \vec{\rho}} \phi_{n}(0) \phi_{n}^{*}(0)}{\epsilon \pm i \eta-\left(k_{x}^{2}+k_{y}^{2}+n \omega_{z}\right)} .
\end{gathered}
$$

Using the form of $\phi_{n}(0)$ and carrying out integrations, one finds

$$
\begin{aligned}
G_{1}^{ \pm}(\rho)= & \sqrt{\frac{\omega_{z}}{2 \pi}} \frac{\mp i\left[J_{0}(\sqrt{\epsilon} \rho) \pm i N_{0}(\sqrt{\epsilon} \rho)\right]}{4}, \\
G_{2}^{ \pm}(\rho)= & -\sqrt{\frac{\omega_{z}}{2 \pi}} \frac{1}{4 \pi} \int_{0}^{\infty} \frac{d t}{t} \exp \left[\frac{-\omega_{z} \rho^{2}}{2 t}+\frac{\epsilon t}{2 \omega_{z}}\right] \\
& \times\left[\frac{1}{\sqrt{1-e^{-t}}}-1\right] .
\end{aligned}
$$

Note that for $G_{2}^{ \pm}$, it is independent of the sign of the infinitesimal imaginary part in the denominator, and we shall denote it as simply $G_{2}$ in the following. Let $\tilde{G}_{2}=\partial G_{2} / \partial \rho$,

$$
\begin{aligned}
\tilde{G}_{2}(\rho)= & \sqrt{\frac{\omega_{z}}{2 \pi}} \frac{\omega_{z} \rho}{4 \pi} \int_{0}^{\infty} \frac{d t}{t^{2}} \exp \left[\frac{-\omega_{z} \rho^{2}}{2 t}+\frac{\epsilon t}{2 \omega_{z}}\right] \\
& \times\left[\frac{1}{\sqrt{1-e^{-t}}}-1\right] .
\end{aligned}
$$

Then the wave function can be written as

$$
\begin{aligned}
\psi= & \sqrt{\frac{\omega_{z}}{2 \pi}} \frac{-i \sqrt{\epsilon}\left[J_{1}(\sqrt{\epsilon} \rho)-i N_{1}(\sqrt{\epsilon} \rho)\right]}{4}+\tilde{G}_{2} \\
& -S\left[\sqrt{\frac{\omega_{z}}{2 \pi}} \frac{i \sqrt{\epsilon}\left[J_{1}(\sqrt{\epsilon} \rho)+i N_{1}(\sqrt{\epsilon} \rho)\right]}{4}+\tilde{G}_{2}\right] \\
\propto & J_{1}(\sqrt{\epsilon} \rho)-\tan \delta_{1} N_{1}(\sqrt{\epsilon} \rho)+\frac{\tan \delta_{1}}{A} \tilde{G}_{2} .
\end{aligned}
$$

Here $A \equiv \sqrt{\frac{\omega_{z}}{2 \pi}} \frac{\sqrt{\epsilon}}{4}$. In the following, we set $k \equiv \sqrt{\epsilon}$. When $\rho^{2} \omega_{z} \ll 1$, the wave function looks like a 3D $p$-wave function.

$$
\begin{aligned}
\psi & \propto j_{1}(p \rho)-\tan \left(\delta^{3 D}\right) n_{1}(p \rho) \\
& \propto \cot \left(\delta^{3 D}\right) j_{1}(p \rho)-n_{1}(p \rho) \\
& \propto\left[-\frac{1}{3 V^{3 D}}-\frac{p^{2}}{3 R^{3 D}}\right](\rho+\cdots)+\left[\frac{1}{\rho^{2}}+\cdots\right] .
\end{aligned}
$$

Here $V^{3 D}$ and $R^{3 D}$ are the $p$-wave scattering volume and effective range in three dimensions, respectively [17]. The energy $E=p^{2}=k^{2}+\omega_{z} / 2$. In the above equation, the effective expansion for the 3D $p$-wave phase shift $p^{3} \cot \left(\delta^{3 D}\right)=$ $-1 / V^{3 D}-p^{2} / R^{3 D}$ has been used. $j_{1}$ and $n_{1}$ are spherical Bessel functions. At small $\rho$, we need to calculate the coefficients of $\rho$ and $\rho^{-2}$ from $J_{1}, N_{1}$, and $\tilde{G}_{2}$ and then compare the above two formulas to get the effective $2 \mathrm{D} p$-wave interaction parameters in terms of 3D $p$-wave parameters $V^{3 D}$ and $R^{3 D}$. As $\rho \rightarrow 0$, the most divergent term is proportional to $1 / \rho^{2}$, which comes from

$$
\begin{aligned}
\tilde{G}_{2} & \sim \sqrt{\frac{\omega_{z}}{2 \pi}} \frac{\omega_{z} \rho}{4 \pi} \int_{0}^{\infty} \frac{d t}{t^{2}} \exp \left[\frac{-\omega_{z} \rho^{2}}{2 t}+\frac{\epsilon t}{2 \omega_{z}}\right] \sqrt{\frac{1}{t}} \\
& \sim \frac{1}{4 \pi \rho^{2}} .
\end{aligned}
$$

As a result, the coefficient of $1 / \rho^{2}$ is $\frac{\tan \delta_{1}}{4 \pi A}$ in Eq. (B12).

Next, we calculate the coefficient of $\rho$. There exist $\rho \ln \rho$ and $1 / \rho$ terms in the expansion of Bessel's function $N_{1}(k \rho)$ near the origin. However, there are no such terms $(\rho \ln \rho$ and $1 / \rho)$ in the $3 \mathrm{D}$ scattering wave function $\left(j_{1}\right.$ and $\left.n_{1}\right)$. In fact, it can be shown that these singular terms from $N_{1}(k r)$ are exactly canceled by those from $\tilde{G}_{2}$. Here we need to isolate these singular terms with a formula:

$$
\begin{aligned}
\Delta \tilde{G}_{2}= & \tilde{G}_{2}-g_{2} \\
= & \sqrt{\frac{\omega_{z}}{2 \pi}} \frac{\omega_{z} \rho}{4 \pi} \int_{0}^{\infty} \frac{d t}{t^{2}} \exp \left[\frac{-\omega_{z} \rho^{2}}{2 t}+\frac{k^{2} t}{2 \omega_{z}}\right]\left[\frac{1}{\sqrt{1-e^{-t}}}\right. \\
& \left.-1-\frac{e^{-t}}{\sqrt{t}}+e^{-t}-\frac{5 \sqrt{t} e^{-t}}{4}+t e^{-t}\right] .
\end{aligned}
$$

Here $g_{2}$ gives all the mainly singular terms, e.g., $1 / \rho^{2}, 1 / \rho, \rho \ln \rho$, occurring in $\tilde{G}_{2}$ as $\rho \rightarrow 0$. Then $\Delta \tilde{G}_{2}$ is not singular compared with the linear term $\rho$ as $\rho \rightarrow 0$, e.g., $\Delta \tilde{G}_{2} / \rho \sim O(1)$. When $\rho \rightarrow 0$, we have

$$
\Delta \tilde{G}_{2}=\sqrt{\frac{\omega_{z}}{2 \pi}} \frac{\omega_{z}}{4 \pi} \beta\left(k^{2} / \omega_{z}\right) \rho
$$


and

$$
\begin{aligned}
\beta\left(k^{2} / \omega_{z}\right) \equiv & \int_{0}^{\infty} \frac{d t}{t^{2}} \exp \left[\frac{k^{2} t}{2 \omega_{z}}\right]\left[\frac{1}{\sqrt{1-e^{-t}}}-1\right. \\
& \left.-\frac{e^{-t}}{\sqrt{t}}+e^{-t}-\frac{5 \sqrt{t} e^{-t}}{4}+t e^{-t}\right] .
\end{aligned}
$$

Collecting all the linear terms of $\rho$ from $J_{1}, N_{1}, g_{2}$, and $\Delta \tilde{G}_{2}$ and comparing the coefficients of $\rho$ and $1 / \rho^{2}$ in Eqs. (B12) and (B13), we can get the effective $p$-wave interaction parameters $a$ and $R$ in two dimensions in terms of $V^{3 D}$ and $R^{3 D}$ :

$$
\begin{gathered}
a=\frac{3 \pi V^{3 D}}{2 \sqrt{2 \pi} l_{z}+\frac{\sqrt{2 \pi} V^{3 D}}{R^{3} l_{z}}+\frac{V^{3 D}}{l_{z}^{2}}[6-7 \sqrt{\pi}+6 \beta(0)]}, \\
R=\frac{e^{\frac{3 \sqrt{\pi}}{8}-\frac{\gamma}{2}}}{\sqrt{2}} e^{-\frac{\sqrt{2 \pi} l_{z}}{3 R^{3 D}}} l_{z},
\end{gathered}
$$

where $l_{z}=\sqrt{\hbar / M \omega_{z}}$ and $\beta(0) \approx 1.01353$. The coefficient $\gamma$ is given by

$$
\begin{aligned}
\gamma \equiv & \int_{0}^{\infty} \frac{d t}{t}\left[\frac{1}{\sqrt{1-e^{-t}}}-1-\frac{e^{-t}}{\sqrt{t}}+e^{-t}\right. \\
& \left.-\frac{5 \sqrt{t} e^{-t}}{4}+t e^{-t}\right],
\end{aligned}
$$

which is approximately 0.3915 .

In the following, one can choose the parameters which are experimentally accessible in ${ }^{40} \mathrm{~K}$ [11]. Consider a harmonic trap with frequency of the order of $\omega_{z}=2 \pi \times 120 \mathrm{kHz}$, and then use

$$
V^{3 D}=V_{b g}\left(1-\frac{\Delta_{V}}{\delta B}\right)
$$

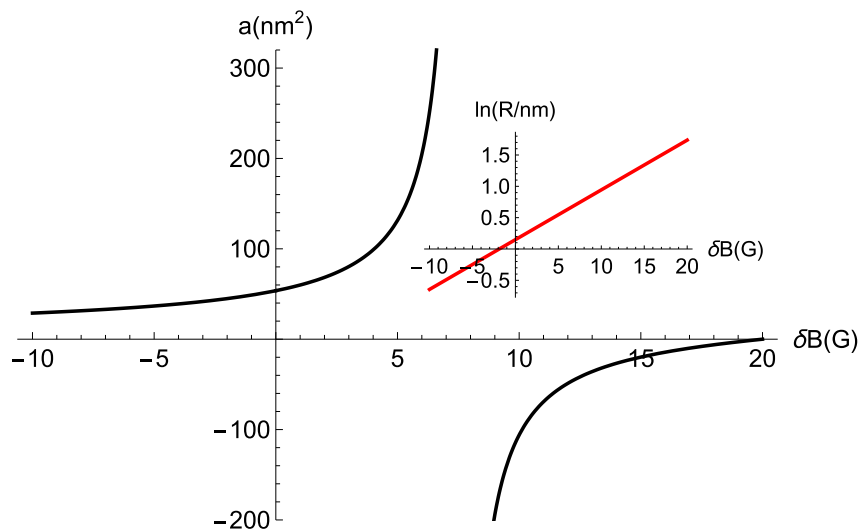

FIG. 4. The scattering area $a$ as a function of magnetic field $\delta B$ (relative to $3 \mathrm{D}$ resonance point). The inset shows the variations of effective range $R$ with magnetic field $\delta B$.

where the (magnetic) width of the resonance is $\Delta_{V}=20 \mathrm{G}$. $V_{b g}=\left(100 a_{0}\right)^{3}$ is the background scattering volume. $a_{0}$ is the Bohr radius. For the effective range, we have

$$
\frac{1}{R^{3 D}}=\frac{1}{R_{b g}}\left(1+\frac{\delta B}{\Delta_{R}}\right)
$$

where $\Delta_{R}=-20 \mathrm{G}$ and $R_{b g}=50 a_{0}$. The above experimental parameters can realize a quasi-2D Fermi gas which satisfies $R^{3 D} \ll l_{z} \ll 1 / k_{F}$ [44]. In order to realize a true 2D Fermi gas where $R^{3 D} \sim l_{z} \ll 1 / k_{F}$ one needs a trap frequency of the order of $\omega_{z} \sim 10 \mathrm{MHz}$. In Fig. 4, for $\omega_{z}=10 \mathrm{MHz}$, we show the variations of the effective 2D scattering area $a$ and the effective range $R$ when magnetic field $\delta B$ (relative to the $3 \mathrm{D}$ resonance point) varies. Note that the resonance position is shifted by the external trap potential.
[1] C. A. Regal, C. Ticknor, J. L. Bohn, and D. S. Jin, Tuning $p$-Wave Interactions in an Ultracold Fermi Gas of Atoms, Phys. Rev. Lett. 90, 053201 (2003).

[2] J. Zhang, E. G. M. van Kempen, T. Bourdel, L. Khaykovich, J. Cubizolles, F. Chevy, M. Teichmann, L. Tarruell, S. J. J. M. F. Kokkelmans, and C. Salomon, $P$-wave Feshbach resonances of ultracold ${ }^{6}$ Li, Phys. Rev. A 70, 030702(R) (2004).

[3] C. Ticknor, C. A. Regal, D. S. Jin, and J. L. Bohn, Multiplet structure of Feshbach resonances in nonzero partial waves, Phys. Rev. A 69, 042712 (2004).

[4] K. Günter, T. Stöferle, H. Moritz, M. Köhl, and T. Esslinger, $p$ Wave Interactions in Low-Dimensional Fermionic Gases, Phys. Rev. Lett. 95, 230401 (2005).

[5] C. H. Schunck, M. W. Zwierlein, C. A. Stan, S. M. F. Raupach, W. Ketterle, A. Simoni, E. Tiesinga, C. J. Williams, and P. S. Julienne, Feshbach resonances in fermionic ${ }^{6} \mathrm{Li}$, Phys. Rev. A 71, 045601 (2005).

[6] J. P. Gaebler, J. T. Stewart, J. L. Bohn, and D. S. Jin, p-Wave Feshbach Molecules, Phys. Rev. Lett. 98, 200403 (2007).

[7] Y. Inada, M. Horikoshi, S. Nakajima, M. Kuwata-Gonokami, M. Ueda, and T. Mukaiyama, Collisional Properties of $p$-Wave Feshbach Molecules, Phys. Rev. Lett. 101, 100401 (2008).
[8] J. Fuchs, C. Ticknor, P. Dyke, G. Veeravalli, E. Kuhnle, W. Rowlands, P. Hannaford, and C. J. Vale, Binding energies of ${ }^{6} \mathrm{Li} p$-wave Feshbach molecules, Phys. Rev. A 77, 053616 (2008).

[9] T. Nakasuji, J. Yoshida, and T. Mukaiyama, Experimental determination of $p$-wave scattering parameters in ultracold ${ }^{6} \mathrm{Li}$ atoms, Phys. Rev. A 88, 012710 (2013).

[10] M. Waseem, Z. Zhang, J. Yoshida, K. Hattori, T. Saito, and T. Mukaiyama, Creation of $p$-wave Feshbach molecules in the selected angular momentum states using an optical lattice, J. Phys. B 49, 204001 (2016).

[11] C. Luciuk, S. Trotzky, S. Smale, Z. Yu, S. Zhang, and J. H. Thywissen, Evidence for universal relations describing a gas with $p$-wave interactions, Nat. Phys. 12, 599 (2016).

[12] S. Tan, Energetics of a strongly correlated Fermi gas, Ann. Phys. (NY) 323, 2952 (2008).

[13] E. Braaten and L. Platter, Exact Relations for a Strongly Interacting Fermi Gas from the Operator Product Expansion, Phys. Rev. Lett. 100, 205301 (2008).

[14] S. Zhang and A. J. Leggett, Universal properties of the ultracold Fermi gas, Phys. Rev. A 79, 023601 (2009). 
[15] F. Werner, L. Tarruell, and Y. Castin, Number of closed-channel molecules in the BEC-BCS crossover, Eur. Phys. J. B 68, 401 (2009).

[16] S. M. Yoshida and M. Ueda, Universal High-Momentum Asymptote and Thermodynamic Relations in a Spinless Fermi Gas with a Resonant $p$-Wave Interaction, Phys. Rev. Lett. 115, 135303 (2015).

[17] Z. Yu, J. H. Thywissen, and S. Zhang, Universal Relations for a Fermi Gas Close to a $p$-Wave Interaction Resonance, Phys. Rev. Lett. 115, 135304 (2015).

[18] M.-Y. He, S.-L. Zhang, H. M. Chan, and Q. Zhou, Concept of Contact Spectrum and Its Applications in Atomic Quantum Hall States, Phys. Rev. Lett. 116, 045301 (2016).

[19] S.-L. Zhang, M. He, and Q. Zhou, Contact matrix in dilute quantum systems, arXiv:1606.05176.

[20] S. M. Yoshida and M. Ueda, $p$-wave contact tensor: Universal properties of axisymmetry-broken $p$-wave Fermi gases, Phys. Rev. A 94, 033611 (2016).

[21] S.-G. Peng, X.-J. Liu, and H. Hu, Large-momentum distribution of a polarized Fermi gas and $p$-wave contacts, Phys. Rev. A 94, 063651 (2016).

[22] X. Cui and H. Dong, High-momentum distribution with a subleading $k^{-3}$ tail in the odd-wave interacting one-dimensional Fermi gases, Phys. Rev. A 94, 063650 (2016).

[23] J. Yao and S. Zhang, Normal state properties of a resonantly interacting p-wave Fermi Gas, arXiv:1609.06476.

[24] L. P. Pitaevskii and A. Rosch, Breathing modes and hidden symmetry of trapped atoms in two dimensions, Phys. Rev. A 55, 853(R) (1997).

[25] M. Randeria, J.-M. Duan, and L.-Y. Shieh, Superconductivity in a two-dimensional Fermi gas: Evolution from Cooper pairing to Bose condensation, Phys. Rev. B 41, 327 (1990).

[26] H.-W. Hammer and D. Lee, Causality and universality in low-energy quantum scattering, Phys. Lett. B 681, 500 (2009).

[27] H.-W. Hammer and D. Lee, Causality and the effective range expansion, Ann. Phys. (NY) 325, 2212 (2010).

[28] S. A. Rakityansky and N. Elander, Analytic structure and power series expansion of the Jost function for the two-dimensional problem, J. Phys. A 45, 135209 (2012).
[29] H. A. Bethe, Theory of the Effective Range in Nuclear Scattering, Phys. Rev. 76, 38 (1949).

[30] L. B. Madsen, Effective range theory, Am. J. Phys. 70, 811 (2002).

[31] Y. Nishida, S. Moroz, and D. T. Son, Super Efimov Effect of Resonantly Interacting Fermions in Two Dimensions, Phys. Rev. Lett. 110, 235301 (2013).

[32] A. G. Volosniev, D. V. Fedorov, A. S. Jensen, and N. T. Zinner, Borromean ground state of fermions in two dimensions, J. Phys. B 47, 185302 (2014).

[33] C. Gao, J. Wang, and Z. Yu, Revealing the origin of super-Efimov states in the hyperspherical formalism, Phys. Rev. A 92, 020504 (2015).

[34] P. Zhang and Z. Yu, Sigature of the universal super Efimov Effect: Three-body contact in two dimensional Fermi gases, arXiv:1611.09454.

[35] T.-L. Ho and E. J. Mueller, High Temperature Expansion Applied to Fermions near Feshbach Resonance, Phys. Rev. Lett. 92, 160404 (2004).

[36] X.-J. Liu, H. Hu, and P. D. Drummond, Virial Expansion for a Strongly Correlated Fermi Gas, Phys. Rev. Lett. 102, 160401 (2009).

[37] K. Huang, Statistical Mechanics 2nd ed. (Wiley, New York, 1987).

[38] J. Hofmann, Quantum Anomaly, Universal Relations, and Breathing Mode of a Two-Dimensional Fermi Gas, Phys. Rev. Lett. 108, 185303 (2012).

[39] C. Gao and Z. Yu, Breathing mode of two-dimensional atomic Fermi gases in harmonic traps, Phys. Rev. A 86, 043609 (2012).

[40] E. Taylor and M. Randeria, Apparent Low-Energy Scale Invariance in Two-Dimensional Fermi Gases, Phys. Rev. Lett. 109, 135301 (2012).

[41] J. Levinsen, Stability of fermionic gases close to a $p$-wave Feshbach resonance, Phys. Rev. A 78, 063616 (2008).

[42] Z. Idziaszek and T. Calarco, Pseudopotential Method for Higher Partial Wave Scattering, Phys. Rev. Lett. 96, 013201 (2006).

[43] S.-G. Peng, S. Tan, and K. Jiang, Manipulation of $p$-Wave Scattering of Cold Atoms in Low Dimensions Using the Magnetic Field Vector, Phys. Rev. Lett. 112, 250401 (2014).

[44] J. H. Thywissen (private communication). 